\title{
Corrigendum: Differential Risk of Dementia Between Patients With Atrial Flutter and Atrial Fibrillation: A National Cohort Study
}

\section{OPEN ACCESS}

Approved by:

Frontiers Editorial Office,

Frontiers Media SA, Switzerland

*Correspondence:

Mien-Cheng Chen

chenmien@ms76.hinet.net

†These authors have contributed equally to this work and share first authorship

$¥$ These authors have contributed equally to this work

Specialty section:

This article was submitted to Cardiac Rhythmology,

a section of the journal

Frontiers in Cardiovascular Medicine

Received: 22 November 2021 Accepted: 24 November 2021

Published: 09 December 2021

Citation:

Wang $H-T$, Chen $Y-L$, Lin Y-S, Chen H-C, Chong S-Z, Hsueh S,

Chung C-M and Chen M-C (2021)

Corrigendum: Differential Risk of Dementia Between Patients With Atrial

Flutter and Atrial Fibrillation: A National Cohort Study.

Front. Cardiovasc. Med. 8:819771. doi: 10.3389/fcvm.2021.819771

\begin{abstract}
Hui-Ting Wang ${ }^{1 \dagger}$, Yung-Lung Chen ${ }^{2,3}$, Yu-Sheng Lin ${ }^{3,4+}$, Huang-Chung Chen ${ }^{2}$, Shaur-Zheng Chong ${ }^{2}$, Shukai Hsueh ${ }^{2}$, Chang-Ming Chung ${ }^{4 \neq}$ and Mien-Cheng Chen ${ }^{2 \star \neq}$

${ }^{1}$ Department of Emergency, Kaohsiung Chang Gung Memorial Hospital, Kaohsiung, Taiwan, ${ }^{2}$ Division of Cardiology, Department of Internal Medicine, Kaohsiung Chang Gung Memorial Hospital, Kaohsiung, Taiwan, ${ }^{3}$ College of Medicine, Graduate Institute of Clinical Medical Sciences, Chang Gung University, Taoyuan, Taiwan, ${ }^{4}$ Division of Cardiology, Department of Internal Medicine, Chang Gung Memorial Hospital, Chiayi, Taiwan
\end{abstract}

Keywords: atrial fibrillation, atrial flutter, dementia, stroke, $\mathrm{CHA}_{2} \mathrm{DS}_{2}$-VASc score

\section{A Corrigendum on}

Differential Risk of Dementia Between Patients With Atrial Flutter and Atrial Fibrillation: A National Cohort Study

by Wang, H.-T., Chen, Y.-L., Lin, Y.-S., Chen, H.-C., Chong, S.-Z., Hsueh, S., Chung, C.-M., and Chen, M.-C. (2021). Front. Cardiovasc. Med. 8:787866. doi: 10.3389/fcvm.2021.787866

In the original article there was an error with the Author Contributions Statement. The corrected Author Contributions Statement appears below.

Hui-Ting Wang and Yu-Sheng Lin contributed equally as first authors

Chang-Ming Chung and Mien-Cheng Chen contributed equally as corresponding authors

The authors apologize for this error and state that this does not change the scientific conclusions of the article in any way. The original article has been updated.

Publisher's Note: All claims expressed in this article are solely those of the authors and do not necessarily represent those of their affiliated organizations, or those of the publisher, the editors and the reviewers. Any product that may be evaluated in this article, or claim that may be made by its manufacturer, is not guaranteed or endorsed by the publisher.

Copyright (c) 2021 Wang, Chen, Lin, Chen, Chong, Hsueh, Chung and Chen. This is an open-access article distributed under the terms of the Creative Commons Attribution License (CC BY). The use, distribution or reproduction in other forums is permitted, provided the original author(s) and the copyright owner(s) are credited and that the original publication in this journal is cited, in accordance with accepted academic practice. No use, distribution or reproduction is permitted which does not comply with these terms. 